\title{
The Łojasiewicz exponent at infinity for overdetermined polynomial mappings
}

\author{
by S. Spodzieja (Łódź)
}

\begin{abstract}
We prove that the study of the Łojasiewicz exponent at infinity of overdetermined polynomial mappings $\mathbb{C}^{n} \rightarrow \mathbb{C}^{m}, m>n$, can be reduced to the one when $m=n$.
\end{abstract}

Introduction. In the paper we study the Eojasiewicz exponent at infinity for overdetermined polynomial mappings, i.e. polynomial mappings $f: \mathbb{C}^{n} \rightarrow \mathbb{C}^{m}$, where $m>n$. In the case $m=n$ this exponent is well known (see $[\mathrm{C}],\left[\mathrm{CK}_{1}\right]-\left[\mathrm{CK}_{4}\right],\left[\mathrm{P}_{1}\right],\left[\mathrm{P}_{2}\right],[\mathrm{PT}]$ ). It is strongly related to the properties of properness and injectivity of polynomial mappings $($ see $[\mathrm{H}],[\mathrm{C}]$, $\left.\left[\mathrm{CK}_{1}\right],\left[\mathrm{CK}_{3}\right],\left[\mathrm{CK}_{4}\right],\left[\mathrm{P}_{1}\right],\left[\mathrm{P}_{2}\right],[\mathrm{PT}]\right)$. Numerous papers have been devoted to the estimation of this exponent from below and to the effective Nullstellensatz (see $[\mathrm{C}],\left[\mathrm{B}_{1}\right],\left[\mathrm{B}_{2}\right],[\mathrm{JKS}],[\mathrm{K}],[\mathrm{S}],[\mathrm{BY}],\left[\mathrm{CK}_{5}\right]$ ). The deepest result in this direction is the Kollár inequality $[\mathrm{K}]$. We investigate it in Corollary 3.2.

We reduce the computation of the exponent of $f: \mathbb{C}^{n} \rightarrow \mathbb{C}^{m}, m>n$, to the case $m=n$ (see Theorem 2.1 and Corollary 3.1). The key point of the proof is the reduction of the study of the fibres of a polynomial mapping to the case of $m \leq n$ (Proposition 1.1). We obtain it by composing $f$ with a linear mapping. This method can be applied to obtain a characterization of the Łojasiewicz exponent of a proper polynomial mapping (Corollary 3.3, cf. $\left.[\mathrm{C}],\left[\mathrm{P}_{1}\right]\right)$. Corollary 3.3 also gives a criterion for injectivity of polynomial mappings (cf. $\left.\left[\mathrm{P}_{1}\right]\right)$.

Additionally, using the Łojasiewicz exponent at infinity we prove a criterion of properness of polynomial mappings (Corollary 3.4, cf. $[\mathrm{C}],\left[\mathrm{P}_{1}\right]$ ).

1. Fibres of polynomial mappings. In what follows we write "the generic $x \in A$ " instead of "there exists an algebraic set $V$ such that $A \backslash V$ is a dense subset of $A$ and $x \in A \backslash V$ ".

2000 Mathematics Subject Classification: Primary 14R99; Secondary 32H35.

Key words and phrases: polynomial mapping, Łojasiewicz exponent at infinity.

This research was partially supported by KBN Grant No. 2 P03A 00718. 
For any $m, k \in \mathbb{N}$ we denote by $\mathbf{L}(m, k)$ the set of all nonsingular linear mappings $\mathbb{C}^{m} \rightarrow \mathbb{C}^{k}$, where for $k=0$ we put $\mathbb{C}^{k}=\{0\}$. Let $m \geq k$. Denote by $\Delta(m, k)$ the set of all linear mappings $L=\left(L_{1}, \ldots, L_{k}\right) \in \mathbf{L}(m, k)$ of the form

$$
L_{i}\left(y_{1}, \ldots, y_{m}\right)=y_{i}+\sum_{j=k+1}^{m} \alpha_{i, j} y_{j}, \quad i=1, \ldots, k,
$$

where $\alpha_{i, j} \in \mathbb{C} ; \Delta^{0}(m, k)$ is the set of all $L=\left(L_{1}, \ldots, L_{k}\right) \in \Delta(m, k)$ such that

$$
L_{1}\left(y_{1}, \ldots, y_{m}\right)=y_{1} .
$$

Proposition 1.1. Let $f=\left(f_{1}, \ldots, f_{m}\right): \mathbb{C}^{n} \rightarrow \mathbb{C}^{m}$ be a polynomial mapping with $\operatorname{deg} f_{j}>0$ for $j=1, \ldots, m$, where $m \geq n \geq 1$.

(i) For the generic $L \in \mathbf{L}(m, n)$,

$$
\#\left[(L \circ f)^{-1}(0) \backslash f^{-1}(0)\right]<\infty .
$$

(ii) For the generic $L \in \Delta^{0}(m, n)$, (1) holds.

The proof will be preceded by an easy lemma. We denote by $G_{k}\left(\mathbb{C}^{m}\right)$ the Grassmann space of $k$-dimensional linear subspaces of $\mathbb{C}^{m}, k<m$.

Lemma 1.1. Let $V \subset \mathbb{C}^{m}$ be an algebraic set of dimension $s$.

(i) If $s+k<m$, then for the generic $H \in G_{k}\left(\mathbb{C}^{m}\right)$,

$$
H \cap V \subset\{0\} .
$$

(ii) If $s+k=m$, then for the generic $H \in G_{k}\left(\mathbb{C}^{m}\right)$,

$$
\#(H \cap V)<\infty .
$$

Proof of Proposition 1.1. In the proof of (ii) we will need a version of (i) in the case of regular mappings. So, we prove (i) in the slightly general case of regular mappings $f=\left(f_{1}, \ldots, f_{m}\right): X \rightarrow \mathbb{C}^{m}$, where $X$ is an irreducible algebraic set, $\operatorname{dim} X \leq n$ and $f_{j} \neq$ const. Let $W \subset \mathbb{C}^{m}$ be the closure of $f(X)$ and $k=\operatorname{dim} W$. Obviously $k \leq n$. We have two cases:

$1^{\circ} . k<n$. By Lemma 1.1(i) there exists a Zariski open and dense subset $U \subset G_{m-n}\left(\mathbb{C}^{m}\right)$ such that for any $H \in U$ we have $W \cap H \subset\{0\}$. Hence, the set $\mathcal{U}=\{L \in \mathbf{L}(m, n)$ : $\operatorname{ker} L \in U\}$ is a Zariski open and dense subset of $\mathbf{L}(m, n)$. Moreover, for any $L \in \mathcal{U}$ we have $f^{-1}(0)=(L \circ f)^{-1}(0)$. This gives (1) in this case.

$2^{\circ} . k=n$. Let

$$
\Gamma=\overline{\left\{w \in W: \operatorname{dim} f^{-1}(w)>0\right\}} .
$$

By Corollary 3.16 and Proposition 2.31 of $[\mathrm{M}], \Gamma$ is an algebraic set. Moreover $\operatorname{dim} \Gamma<n$, since in the opposite case, by the definition of $\Gamma$ and 
Corollary 3.15 of $[\mathrm{M}]$, we have

$$
n+1 \leq \operatorname{dim} f^{-1}(\Gamma) \leq \operatorname{dim} f^{-1}(W)=n,
$$

which is impossible. Thus, by Lemma 1.1, there exists a Zariski open and dense subset $U \subset G_{m-n}\left(\mathbb{C}^{m}\right)$ such that for any $H \in U$ we have

$$
\#(W \cap H)<\infty
$$

and

$$
\Gamma \cap H \subset\{0\}
$$

Let, by (2), $W \cap H=\left\{w^{1}, \ldots, w^{p}\right\}$ and $L \in \mathbf{L}(m, n)$ be such that $H=\operatorname{ker} L$. Then

$$
(L \circ f)^{-1}(0)=f^{-1}\left(w^{1}\right) \cup \ldots \cup f^{-1}\left(w^{p}\right) .
$$

From (3) it follows that $w^{i} \notin \Gamma$ if $w^{i} \neq 0$. In consequence for $w^{i} \neq 0$ we have $\# f^{-1}\left(w^{i}\right)<\infty$. This gives (1). Since $\{L \in \mathbf{L}(m, n): \operatorname{ker} L \in U\}$ is a Zariski open and dense subset of $\mathbf{L}(m, n)$, we have the assertion in this case. This gives (i).

To prove (ii), let $X^{\prime}=f^{-1}\left(\{0\} \times \mathbb{C}^{m-1}\right)$ and $g: X^{\prime} \rightarrow \mathbb{C}^{m-1}$ be a regular mapping of the form

$$
g(x)=\left(f_{2}(x), \ldots, f_{m}(x)\right), \quad x \in X^{\prime} .
$$

Since $f_{1} \neq 0, \operatorname{dim} X^{\prime} \leq n-1$. From the first part of the proof, we now see that for the generic $M \in \mathbf{L}(m-1, n-1)$,

$$
\#\left[(M \circ g)^{-1}(0) \backslash g^{-1}(0)\right]<\infty .
$$

Obviously the set $\mathcal{U}$ of all linear mappings $M=\left(L_{2}, \ldots, L_{n}\right) \in \mathbf{L}(m-1$, $n-1)$ of the form $L_{i}\left(w_{2}, \ldots, w_{m}\right)=L_{i}^{\prime}\left(w_{2}, \ldots, w_{n}\right)+L_{i}^{\prime \prime}\left(w_{n+1}, \ldots, w_{m}\right)$, $i=2, \ldots, n$, such that $\operatorname{Jac}\left[L_{2}^{\prime}, \ldots, L_{n}^{\prime}\right] \neq 0$ is a Zariski open and dense subset of $\mathbf{L}(m-1, n-1)$. Moreover, $\left(L_{2}^{\prime}, \ldots, L_{n}^{\prime}\right)^{-1} \circ M \in \Delta(m-1, n-1)$. So, for the generic $M \in \Delta(m-1, n-1)$ we have (4). Since $L=\left(w_{1}, M\right) \in \Delta^{0}(m, n)$ for any $M \in \mathcal{U}$, we obtain (ii).

2. The Kojasiewicz exponent at infinity. In this section we prove Theorem 2.1 on reduction of calculations of the Eojasiewicz exponent at infinity of a mapping $\mathbb{C}^{n} \rightarrow \mathbb{C}^{m}$ to the case $\mathbb{C}^{n} \rightarrow \mathbb{C}^{n}$.

Let $f: \mathbb{C}^{n} \rightarrow \mathbb{C}^{m}$ be a polynomial mapping such that $\# f^{-1}(0)<\infty$. Let

$$
N_{\infty}(f)=\left\{\nu \in \mathbb{R}: \exists_{C>0, R>0} \forall_{x \in \mathbb{C}^{n}}|x|>R \Rightarrow|f(x)| \geq C|x|^{\nu}\right\},
$$

where $|\cdot|$ denotes the policylindric norm. We define the Eojasiewicz exponent at infinity of the mapping $f$ as $\sup N_{\infty}(f)$ and denote it by $\mathcal{L}_{\infty}(f)$.

ThEOREM 2.1. Let $f: \mathbb{C}^{n} \rightarrow \mathbb{C}^{m}, m \geq n$, be a polynomial mapping such that $\# f^{-1}(0)<\infty$. Then for any $L \in \mathbf{L}(m, n)$ such that $\#(L \circ f)^{-1}(0)<\infty$ 
we have

$$
\mathcal{L}_{\infty}(f) \geq \mathcal{L}_{\infty}(L \circ f) .
$$

Moreover, for the generic $L \in \mathbf{L}(m, n)$,

$$
\mathcal{L}_{\infty}(f)=\mathcal{L}_{\infty}(L \circ f) .
$$

The proof of this theorem will be preceded by two lemmas.

LEMMA 2.1. Let $V \subset \mathbb{C}^{m}$ be an algebraic set of dimension $s$. If $s<n$, then there exists a Zariski open and dense subset $U \subset \mathbf{L}(m, n)$ such that for any $L \in U$ and for any $\varepsilon>0$ there exists $\delta>0$ such that for any $y \in V$,

$$
|L(y)|<\delta \Rightarrow|y|<\varepsilon .
$$

Proof. There exists a Zariski open and dense subset $U_{1} \subset \mathbf{L}(m, n)$ such that for any $L \in U_{1}$,

$$
V \cap \operatorname{ker} L \subset\{0\}
$$

(see Lemma 1.1(i)). By Sadullaev's Theorem ([€], VII,7.1), there exists a Zariski open and dense subset $U \subset U_{1}$ such that for any $L \in U$ there exists $C_{L}>0$ such that $V \subset\left\{y \in \mathbb{C}^{m}:|y| \leq C_{L}(1+|L(y)|)\right\}$, which implies

$$
y \in V \wedge|y|>2 C_{L} \Rightarrow|L(y)|>1 \text {. }
$$

Let $L \in U$ and $\varepsilon>0$. If $0 \notin V$, then either $A_{1}=\left\{|L(y)|: y \in V \wedge|y| \leq 2 C_{L}\right\}$ is an empty set and we put $\delta_{1}=1$, or $A_{1} \neq \emptyset$ and we put $\delta_{1}=\min A_{1}$. By (7) we have $\delta_{1}>0$. Putting $\delta=\min \left(\delta_{1}, 1\right)$, by (8) and the definition of $A_{1}$ we obtain $|L(y)| \geq \delta$ for any $y \in V$. This gives the assertion in this case. If $0 \in V$, then either $A_{2}=\left\{|L(y)|: y \in V \wedge \varepsilon \leq|y| \leq 2 C_{L}\right\}$ is an empty set and we put $\delta_{2}=1$, or $A_{2} \neq \emptyset$ and we put $\delta_{2}=\min A_{2}$. By (7) we have $\delta_{2}>0$. Putting $\delta=\min \left(\delta_{2}, 1\right)$, by (8) and the definition of $A_{2}$ we obtain the assertion in this case. This ends the proof.

LEMMA 2.2. Let $f: \mathbb{C}^{n} \rightarrow \mathbb{C}^{m}$ with $m \geq n$ be a polynomial mapping. Then there exists a Zariski open and dense subset $U \subset \mathbf{L}(m, n)$ such that for any $L \in U$ and any $\varepsilon>0$ there exist $\delta>0$ and $r>0$ such that for any $x \in \mathbb{C}^{n}$,

$$
|x|>r \wedge|L \circ f(x)|<\delta \Rightarrow|f(x)|<\varepsilon .
$$

Proof. Let $W=\overline{f\left(\mathbb{C}^{n}\right)}$. Then $\operatorname{dim} W \leq n$. Assume first that $\operatorname{dim} W<n$. Then, by Lemma 2.1, there exists a Zariski open and dense subset $U \subset$ $\mathbf{L}(m, n)$ such that for any $L \in U$ and any $\varepsilon>0$ there exists $\delta>0$ such that for any $y \in W$,

$$
|L(y)|<\delta \Rightarrow|y|<\varepsilon
$$

Then for any $x \in \mathbb{C}^{n}$,

$$
|L \circ f(x)|<\delta \Rightarrow|f(x)|<\varepsilon .
$$

Thus we have the assertion in this case. 
Let now $\operatorname{dim} W=n$. Then, by Proposition 3.15 of $[\mathrm{M}]$, we easily see that there exists an algebraic set $V \subset W$ such that $\operatorname{dim} V \leq n-1$ and the mapping

$$
\left.f\right|_{\mathbb{C}^{n} \backslash f^{-1}(V)}: \mathbb{C}^{n} \backslash f^{-1}(V) \rightarrow W \backslash V
$$

is a finite covering. Thus it is a proper mapping. By Lemma 2.1, there exists a Zariski open and dense subset $U_{1} \subset \mathbf{L}(m, n)$ such that for any $L \in U_{1}$ and any $\varepsilon>0$ there exists $\delta>0$ such that for any $y \in V$,

$$
|L(y)|<\delta \Rightarrow|y|<\varepsilon .
$$

By Sadullaev's Theorem there exists a Zariski open and dense subset $U_{2} \subset$ $\mathbf{L}(m, n)$ such that for any $L \in U_{2}$ there exists $C_{L}>0$ such that

$$
W \subset\left\{y \in \mathbb{C}^{m}:|y| \leq C_{L}(1+|L(y)|)\right\} .
$$

Take any $L \in U_{1} \cap U_{2}$ and $\varepsilon>0$. Assume to the contrary that there exists a sequence $\left\{x_{n}\right\}$ such that $\left|x_{n}\right| \rightarrow \infty,\left|L\left(f\left(x_{n}\right)\right)\right| \rightarrow 0$ and

$$
\left|f\left(x_{n}\right)\right| \geq \varepsilon \text {. }
$$

Without loss of generality, by (11), we may assume that $f\left(x_{n}\right) \rightarrow y_{0}$, where $y_{0} \in \mathbb{C}^{m}$. Since the mapping (9) is proper, we see that $y_{0} \in V$. So, $\left|y_{0}\right| \geq \varepsilon$ and $L\left(y_{0}\right)=0$. This contradicts (10) and ends the proof.

Proof of Theorem 2.1. Let

$$
U=\left\{L \in \mathbf{L}(m, n): \#\left[(L \circ f)^{-1}(0)\right]<\infty\right\} .
$$

Let $L \in U$. Then there exists $M \in \mathbf{L}(m, m-n)$ such that $\widetilde{L}=(L, M) \in$ $\mathbf{L}(m, m)$ and $\mathcal{L}_{\infty}(f)=\mathcal{L}_{\infty}(\widetilde{L} \circ f)$. Obviously for $x \in \mathbb{C}^{n}$ we have $|\widetilde{L} \circ f(x)| \geq$ $|L \circ f(x)|$, so $\mathcal{L}_{\infty}(\widetilde{L} \circ f) \geq \mathcal{L}_{\infty}(L \circ f)$. This gives $(5)$.

To prove the "moreover" part, let $W=\overline{f\left(\mathbb{C}^{n}\right)}$. Consider two cases: $\mathcal{L}_{\infty}(f)>0$ and $\mathcal{L}_{\infty}(f) \leq 0$.

First, assume that $\mathcal{L}_{\infty}(f)>0$. Since $\operatorname{dim} W \leq n$, Sadullaev's Theorem yields a Zariski open and dense subset $U_{1} \subset \mathbf{L}(m, n)$ such that for any $L \in U_{1}$ there exist $r>0$ and $M \in \mathbf{L}(m, m-n)$ such that $(L, M) \in \mathbf{L}(m, n)$ and for any $y \in W$,

$$
|y| \geq r \Rightarrow|M(y)| \leq|L(y)|
$$

So,

$$
|y|>r \Rightarrow|(L, M)(y)|=|L(y)| .
$$

By Proposition 1.1(i), $U \cap U_{1}$ contains a Zariski open and dense subset of $\mathbf{L}(m, n)$. Let $L \in U \cap U_{1}$ and $M \in \mathbf{L}(m, m-n)$ be as above. Since $\mathcal{L}_{\infty}(f)>0$, there exists $R_{1}>0$ such that $|f(x)|>r$ for any $x \in \mathbb{C}^{n}$ with $|x|>R_{1}$. Then, from (12),

$$
|x|>R_{1} \Rightarrow|(L, M) \circ f(x)|=|L \circ f(x)| .
$$


Thus, $\mathcal{L}_{\infty}(L \circ f)=\mathcal{L}_{\infty}((L, M) \circ f)$. Since $(L, M)$ is a linear automorphism, we have $\mathcal{L}_{\infty}((L, M) \circ f)=\mathcal{L}_{\infty}(f)$, so we obtain (6) in this case.

Consider the case $\mathcal{L}_{\infty}(f) \leq 0$. If $0 \notin W$, then $\mathcal{L}_{\infty}(f)=0$ and, by Lemma 2.2, for the generic $L \in \mathbf{L}(m, n)$, there exist $\delta>0$ and $r>0$ such that $|L \circ f(x)| \geq \delta$ for any $x \in \mathbb{C}^{n}$ with $|x|>r$. Thus $\mathcal{L}_{\infty}(L \circ f)=0$. This gives (6) in this case. Now, let $0 \in W$ and let $C_{0}(W)$ be the tangent cone to $W$ at $0 \in \mathbb{C}^{m}$ (see [W], p. 510). By Sadullaev's Theorem, there exists a Zariski open and dense subset $U_{2} \subset \mathbf{L}(m, n)$ such that for any $L \in U_{2}$ there exists $M \in \mathbf{L}(m, m-n)$ such that $(L, M) \in \mathbf{L}(m, m)$ and for any $y \in C_{0}(W)$,

$$
|M(y)| \leq \frac{1}{2}|L(y)| .
$$

Thus there exists $\varepsilon>0$ such that for any $y \in W$ with $|y|<\varepsilon$,

$$
|M(y)| \leq|L(y)| \text {. }
$$

Hence, by Lemma 2.2, for $L \in U_{2} \cap U$ there exist $\delta>0$ and $r>0$ such that for any $x \in \mathbb{C}^{n}$ with $|x|>r$ we have

$$
|L \circ f(x)|<\delta \Rightarrow|f(x)|<\varepsilon,
$$

so, there exists $M \in \mathbf{L}(m, m-n)$ such that $(L, M) \in \mathbf{L}(m, m)$ and

$$
|L \circ f(x)|<\delta \Rightarrow|(L, M) \circ f(x)|=|L \circ f(x)| .
$$

Thus, since $\mathcal{L}_{\infty}(f) \leq 0$, we have $\mathcal{L}_{\infty}(L \circ f)=\mathcal{L}_{\infty}((L, M) \circ f)$. Since $(L, M)$ is a linear automorphism, it follows that $\mathcal{L}_{\infty}(f)=\mathcal{L}_{\infty}((L, M) \circ f)$, so we have (6) in this case.

This ends the proof.

3. Corollaries. From Theorem 2.1 we easily obtain the following corollary.

Corollary 3.1. Let $f=\left(f_{1}, \ldots, f_{m}\right): \mathbb{C}^{n} \rightarrow \mathbb{C}^{m}$ be a polynomial mapping, where $m \geq n \geq 1$ and $\# f^{-1}(0)<\infty, d_{j}=\operatorname{deg} f_{j}>0$,

$$
d_{2} \geq \ldots \geq d_{m} \geq d_{1} \text {. }
$$

Then for the generic $L=\left(L_{1}, \ldots, L_{n}\right) \in \Delta^{0}(m, n)$ we have

$$
\begin{gathered}
\operatorname{deg} L_{j} \circ f=d_{j} \quad \text { for } j=1, \ldots, n, \\
\#(L \circ f)^{-1}(0)<\infty, \\
\mathcal{L}_{\infty}(f) \geq \mathcal{L}_{\infty}(L \circ f) .
\end{gathered}
$$

Proof. By Proposition 1.1(ii) and Theorem 2.1, for the generic $L \in$ $\Delta^{0}(m, n)$ we have (14) and (15). By the assumption on the degrees $d_{j}$ and the definition of $\Delta^{0}(m, n)$, for the generic $L \in \Delta^{0}(m, n)$ we have (13). This ends the proof. 
For $\left(d_{1}, \ldots, d_{m}\right) \in \mathbb{Z}^{m}$, define

$$
B\left(n ; d_{1}, \ldots, d_{m}\right)= \begin{cases}d_{1} \ldots d_{m} & \text { if } m \leq n, \\ d_{1} \ldots d_{n-1} d_{m} & \text { if } m>n .\end{cases}
$$

Proposition 1.10 of $[\mathrm{K}]$ gives immediately

Kollár inequality. Let $f=\left(f_{1}, \ldots, f_{m}\right): \mathbb{C}^{n} \rightarrow \mathbb{C}^{m}$ be a polynomial mapping such that $\#\left(f^{-1}(0)\right)<\infty$. Let $d_{j}=\operatorname{deg} f_{j}, d_{1} \geq \ldots \geq d_{m}>0$. Then

$$
\mathcal{L}_{\infty}(f) \geq d_{m}-B\left(n ; d_{1}, \ldots, d_{m}\right) .
$$

For $m=n=2$ this inequality was obtained by Chądzyński [C]. The proof of Proposition 1.10 in $[\mathrm{K}]$ is based on Proposition 4.1 of $[\mathrm{K}]$, where it is assumed that $m \leq n$. Reduction of the case $m>n$ to the case $m \leq n$ is not clearly explained. Corollary 3.1 gives us such a reduction:

COROLlary 3.2. Under the assumptions of the Kollár inequality, if (*) holds for $m \leq n$, then it also holds for $m>n$.

Proof. For $m>n$ the inequality $(*)$ follows from Corollary 3.1 and from (*) for $m \leq n$.

Let us give some corollaries on proper polynomial mappings.

Corollary $3.3\left(\left[\mathrm{P}_{1}\right]\right.$, for $\left.m=n\right)$. Let $f=\left(f_{1}, \ldots, f_{m}\right): \mathbb{C}^{n} \rightarrow \mathbb{C}^{m}$ be a polynomial mapping with $d_{i}=\operatorname{deg} f_{i}, d_{1} \geq \ldots \geq d_{m}>0$. If $f$ is a proper mapping, then

$$
\mathcal{L}_{\infty}(f) \geq d_{m} / B\left(n ; d_{1}, \ldots, d_{m}\right)
$$

If

$$
\mathcal{L}_{\infty}(f)=d_{m} / B\left(n ; d_{1}, \ldots, d_{m}\right),
$$

then $f$ is injective, and so $\mathbb{C}\left[f_{1}, \ldots, f_{m}\right]=\mathbb{C}\left[x_{1}, \ldots, x_{n}\right]$.

Proof. From the assumption we have $m \geq n$. Since $f$ is a proper mapping, it is well known that $\mathcal{L}_{\infty}(f)>0$ (see $\left[\mathrm{CK}_{1}\right]$, Corollary 2). From Theorem 2.1 it follows that there exists $L=\left(L_{1}, \ldots, L_{n}\right) \in \Delta(m, n)$ such that

$$
\begin{gathered}
\operatorname{deg} L_{j} \circ f=d_{j}, \quad j=1, \ldots, n, \\
\mathcal{L}_{\infty}(f)=\mathcal{L}_{\infty}(L \circ f) .
\end{gathered}
$$

Then $\mathcal{L}_{\infty}(L \circ f)>0$, and so $L \circ f$ is a proper mapping. Thus, by (18) and Corollary 1.13 of $\left[\mathrm{P}_{1}\right]$ we have

$$
\mathcal{L}_{\infty}(L \circ f) \geq \frac{1}{d_{1} \ldots d_{n-1}}=\frac{d_{m}}{B\left(n ; d_{1}, \ldots, d_{m}\right)} .
$$

Now (19) gives (16). 
Assume that (17) holds. Then, by (19),

$$
\mathcal{L}_{\infty}(L \circ f)=\frac{d_{n}}{d_{1} \ldots d_{n}},
$$

so, by Corollary 1.13 of $\left[\mathrm{P}_{1}\right], L \circ f: \mathbb{C}^{n} \rightarrow \mathbb{C}^{n}$ is a polynomial automorphism. This shows that $f$ is injective, completing the proof.

REMARK 3.1. Let $f=\left(f_{1}, \ldots, f_{m}\right): \mathbb{C}^{n} \rightarrow \mathbb{C}^{m}$ be a polynomial mapping with $d_{i}=\operatorname{deg} f_{i}, d_{1} \geq \ldots \geq d_{m}>0$. If

$$
\mathcal{L}_{\infty}(f)<\frac{d_{m}}{B\left(n ; d_{1}, \ldots, d_{m}\right)},
$$

then $\mathcal{L}_{\infty}(f) \leq 0$. Indeed, by Corollary $3.3, f$ is not a proper mapping. So, by Corollary 2 of $\left[\mathrm{CK}_{1}\right], \mathcal{L}_{\infty}(f) \leq 0$.

Corollary 3.4 ( $[\mathrm{C}]$ for $n=m=2$, $\left[\mathrm{P}_{1}\right]$ for $\left.m=n\right)$. Let $f=$ $\left(f_{1}, \ldots, f_{m}\right): \mathbb{C}^{n} \rightarrow \mathbb{C}^{m}$ be a polynomial mapping with $d_{i}=\operatorname{deg} f_{i}, d_{1} \geq$ $\ldots \geq d_{m}>0$. If

$$
B\left(n ; d_{1}, \ldots, d_{m}\right)-d_{m}<\# f^{-1}(0)<\infty,
$$

then $f$ is a proper mapping. In particular the ring extension $\mathbb{C}\left[f_{1}, \ldots, f_{m}\right] \subset$ $\mathbb{C}\left[x_{1}, \ldots, x_{n}\right]$ is integral.

Proof. From the assumption we have $m \geq n$. By Corollary 3.1, there exists $L=\left(L_{1}, \ldots, L_{n}\right) \in \mathbf{L}(m, n)$ such that

$$
\begin{gathered}
\operatorname{deg} L_{j} \circ f=d_{j}, \quad j=1, \ldots, n-1, \quad \operatorname{deg} L_{n} \circ f=d_{m}, \\
\# f^{-1}(0) \leq \#(L \circ f)^{-1}(0)<\infty \\
\mathcal{L}_{\infty}(f) \geq \mathcal{L}_{\infty}(L \circ f) .
\end{gathered}
$$

By $(21)$ and the assumption, $B\left(n ; d_{1}, \ldots, d_{m}\right)-d_{m}<\#(L \circ f)^{-1}(0)<\infty$, so $L \circ f: \mathbb{C}^{n} \rightarrow \mathbb{C}^{n}$ is a dominating mapping and for the generic $y \in \mathbb{C}^{n}$,

$$
B\left(n ; d_{1}, \ldots, d_{m}\right)-d_{m}<\#(L \circ f)^{-1}(y)<\infty .
$$

Hence, from (20) and Proposition 1.3 of $\left[\mathrm{P}_{1}\right]$ it follows that the mapping $L \circ f$ is proper. Thus, from Corollary $3.3, \mathcal{L}_{\infty}(L \circ f)>0$. So, by $(22), \mathcal{L}_{\infty}(f)>0$. In consequence $f$ is a proper mapping. The second assertion is an algebraic equivalent of the first one.

REMARK 3.2. Let $f=\left(f_{1}, \ldots, f_{m}\right): \mathbb{C}^{n} \rightarrow \mathbb{C}^{m}$ be a polynomial mapping with $d_{i}=\operatorname{deg} f_{i}, d_{1} \geq \ldots \geq d_{m}>0$. From Corollaries 3.3 and 3.4 we see that if $\max _{j=1, \ldots, m} d_{j}>1$ and

$$
B\left(n ; d_{1}, \ldots, d_{m}\right)-d_{m}<\# f^{-1}(0)<\infty,
$$

then

$$
\mathcal{L}_{\infty}(f)>d_{m} / B\left(n ; d_{1}, \ldots, d_{m}\right)
$$


Acknowledgments. I am deeply grateful to Professors Jacek Chądzyński and Tadeusz Krasiński for their valuable comments and advice.

\section{References}

[ATW] R. Achilles, P. Tworzewski and T. Winiarski, On improper isolated intersection in complex analytic geometry, Ann. Polon. Math. 51 (1990), 21-36.

[BY] C. A. Berenstein et A. Yger, Une formule de Jacobi et ses conséquences, Ann. Sci. École Norm. Sup. 24 (1991), 363-377.

[B $\left.\mathrm{B}_{1}\right] \quad$ W. D. Brownawell, Bounds for the degree in Nulstellensatz, Ann. of Math. 126 (1987), 577-592.

$\left[\mathrm{B}_{2}\right] \quad-$, Distance to common zeros and lower bounds for polynomials, in: Approximations diophantiennes et nombers transcendants (Luminy, 1990), de Gruyter, Berlin, 1992, 51-60.

[C] J. Chądzyński, On proper polynomial mappings, Bull. Acad. Polon. Math. 31 (1983), 115-120.

[CK 1$]$ J. Chądzyński and T. Krasiński, A set on which the Eojasiewicz exponent at infinity is attained, Ann. Polon. Math. 67 (1997), 191-197.

$\left[\mathrm{CK}_{2}\right]-$ - - Exponent of growth of polynomial mappings of $\mathbb{C}^{2}$ into $\mathbb{C}^{2}$, in: Singularities, S. Łojasiewicz (ed.), Banach Center Publ. 20, PWN, Warszawa, 1988, $147-160$.

[CK 3$]$ - - - Sur l'exponent de Eojasiewicz à l'infini pour les applications polynomiales de $\mathbb{C}^{2}$ dans $\mathbb{C}^{2}$ et les composantes des automorphismes polynomiaux de $\mathbb{C}^{2}$, C. R. Acad. Sci. Paris Sér. I Math. 315 (1992), 1399-1402.

$\left[\mathrm{CK}_{4}\right]-$ - - On the Lojasiewicz exponent at infinity for polynomial mappings from $\mathbb{C}^{2}$ to $\mathbb{C}^{2}$ and components of polynomial automorphisms, Ann. Polon. Math. 57 (1992), 291-302.

[CK $\mathrm{CK}_{5}-$ - - On Kollár's type estimations of polynomial mappings, Univ. Iagel. Acta Math. 37 (1999), 69-74.

$[\mathrm{H}] \quad$ L. Hörmander, On the division of distribution by polynomials, Ark. Mat. 3 (1958), 555-568.

[JKS] S. Ji, J. Kollár and B. Shiffman, A global Eojasiewicz inequality for algebraic varieties, Trans. Amer. Math. Soc. 329 (1992), 813-818.

[K] J. Kollár, Sharp effective Nullstellensatz, J. Amer. Math. Soc. 1 (1988), 963-975.

[€] S. Łojasiewicz, Introduction to Complex Analytic Geometry, Birkhäuser, Basel, 1991.

[M] D. Mumford, Algebraic Geometry I. Complex Projective Varieties, Springer, Berlin, 1976.

$\left[\mathrm{P}_{1}\right] \quad$ A. Płoski, On the growth of proper polynomial mappings, Ann. Polon. Math. 45 (1985), 297-309.

$\left[\mathrm{P}_{2}\right] \quad-$, A note on the Lojasiewicz exponent at infinity, Bull. Soc. Sci. Lettres Łódź 44 (1994), 1-15.

[PT] A. Płoski and P. Tworzewski, A separation condition for polynomial mappings, Bull. Polish Acad. Sci. Math. 44 (1996), 327-331.

[S] B. Shiffman, Degree bounds for the division problem in polynomial ideals, Michigan Math. J. 36 (1989), 163-171. 
[W] H. Whitney, Tangents to an analytic variety, Ann. of Math. 81 (1965), 496-549.

Faculty of Mathematics

University of Łódź

Banacha 22

90-238 Łódź, Poland

E-mail: spodziej@imul.uni.lodz.pl

Reçu par la Rédaction le 30.4.1999

Révisé le 21.12.2000 et 28.9.2001 